East Asian Mathematical Journal Vol. 29 (2013), No.3, pp. 259-268

http://dx.doi.org/10.7858/eamj.2013.017

\title{
SOME RESULTS ON KRONECKER PRODUCTS AND COMMUTATION MATRICES
}

\author{
Yong SiK Yun AND Chul KanG*
}

\begin{abstract}
There are a lot of unsolved problems or issues regarding Kronecker products, vec-operator and Commutation matrices. We derived further properties and results of Kronecker products, vec-operator and Commutation Matrices.
\end{abstract}

\section{Introduction}

The central property of the Commutation matrix $I_{m, n}$ is that it transforms $\operatorname{vec} A$ into $\operatorname{vec} A^{\prime}$, where $A$ is an arbitrary $m \times n$ matrix. We shall denote this matrix as $I_{m, n}$. It is known that $I_{m, n}$ can be used for reversing the order of Kronecker product, a property very uesful in the calculation of matrix derivatives and moment of matrix quadratic form.

In section 2, we review a number of known results on Kronecker products, vec-operator and Commutation matrices. In section 3, we shall derive further properties and results of Kronecker products, vec-operator and Commutation Matrices.

\section{Basic Notations and Preliminary Results}

In this section, we summarize some preliminary results on Kronecker products, vec-operator and Commutation matrices.

Definition 1. Let $A=\left(a_{i j}\right)$ be an $m \times n$ matrix and $B=\left(b_{k l}\right)$ be a $p \times q$ matrix. The $m p \times n q$ matrix with $a_{i j} b_{k l}$ as the element in the $(i k)$ th row and the $(j l)$ th column is called Kronecker product of $A$ and $B$ and is denoted by

Received Januaryl 11, 2013; Accepted April 15, 2013

2000 Mathematics Subject Classification. 11A11.

Key words and phrases. Commutation matrix, Kronecker product, vec-operator.

This work was supported by a research grant from Academic Research Foundation of Hankyong National University for a schlarly exchange program in 2012.

*Corresponding author. 
$A \otimes B$; that is,

$$
A \otimes B=\left(\begin{array}{cccc}
a_{11} B & a_{12} B & \cdots & a_{1 n} B \\
a_{21} B & a_{22} B & \cdots & a_{2 n} B \\
\vdots & \vdots & \ddots & \vdots \\
a_{m 1} B & a_{m 2} B & \cdots & a_{m n} B
\end{array}\right) .
$$

Definition 2. For an $m \times n$ matrix $A$, let vec $A$ denote the $m n$ vector obtained by 'vectorizing' $A$; that is,

$$
\operatorname{vec} A=\left(a_{1}^{\prime}, a_{2}^{\prime}, \cdots, a_{n}^{\prime}\right)^{\prime}
$$

if $A=\left(a_{1}, a_{2}, \cdots, a_{n}\right)$, where $a_{i}$ is an $m$ vector.

Definition 3. The Commutation matrix $I_{m, n}$ is an $m n \times m n$ matrix containing $m n$ blocks of order $m \times n$ such that the $(i j)$ th block has a 1 in its $(j i)$ th position and zeroes elsewhere. One has

$$
I_{m, n}=\sum_{i=1}^{n} \sum_{j=1}^{m}\left(H_{i j} \otimes H_{i j}^{\prime}\right)
$$

where $H_{i j}$ is an $n \times m$ matrix with 1 in its $(i j)$ th position and zeroes elsewhere, and can be written as $H_{i j}=e_{i} e_{j}^{\prime}$ is the $i$ th unit column vector of order $n$.

Some preliminary results on Kronecker products, vec operator and Commutation matrices are:

For $I_{m}: m \times m$ identity matrix,

$$
\begin{gathered}
I_{m} \otimes I_{n}=I_{m n} . \\
I_{m, n} I_{n, m}=I_{m n} .
\end{gathered}
$$

Therefore,

$$
\left(I_{m, n}\right)^{-1}=\left(I_{m, n}\right)^{\prime}=I_{n, m} .
$$

For $A: m \times n$ matrix and $B: p \times q$ matrix,

$$
\operatorname{vec}(A \otimes B)=\left(I_{n} \otimes I_{m, q} \otimes I_{p}\right)(\operatorname{vec} A \otimes \operatorname{vec} B) .
$$

For $A, B, C$, and $D$ conformable matrices,

$$
(A B) \otimes(C D)=(A \otimes C)(B \otimes D) .
$$

For details, see Magnus and Neudecker (1979), Neudecker and Wansbeek (1983) and Neudecker and Wansbeek (1987).

\section{Some Results on Kronecker products and Commutation matrices}

In this section, we will show a couple of relation between Kronecker products, vec-operator and Commutation matrices. Those relations are very useful in calculating moment of matrix quadratic form in multivariate analysis. They are also statistically meaningful in matrix algebra.

Theorem 3.1. $I_{m n q} \otimes I_{p, s} \otimes I_{r}=I_{n} \otimes I_{m, q} \otimes I_{p r s}$. 
Proof. Let $A: m \times n, B: p \times q$ and $C: r \times s$ be arbitrary matrices. Applying (4), (5) and (1), Tracy and Sultan (1993) have obtained the following equality.

$$
\begin{aligned}
& \operatorname{vec}(A \otimes B \otimes C) \\
= & \left(I_{n} \otimes I_{m, q s} \otimes I_{p r}\right)\left(I_{m n q} \otimes I_{p, s} \otimes I_{r}\right)(\operatorname{vec} A \otimes \operatorname{vec} B \otimes \operatorname{vec} C) .
\end{aligned}
$$

Also, applying (4), (5) and (1), we get

$$
\begin{aligned}
& \operatorname{vec}(A \otimes B \otimes C) \\
= & \operatorname{vec}((A \otimes B) \otimes C) \\
= & \left(I_{n q} \otimes I_{m p, s} \otimes I_{r}\right)(\operatorname{vec}(A \otimes B) \otimes \operatorname{vec} C) \\
= & \left(I_{n q} \otimes I_{m p, s} \otimes I_{r}\right)\left(\left(I_{n} \otimes I_{m, q} \otimes I_{p}\right)(\operatorname{vec} A \otimes \operatorname{vec} B) \otimes I_{r s} \operatorname{vec} C\right) \\
= & \left(I_{n q} \otimes I_{m p, s} \otimes I_{r}\right)\left(I_{n} \otimes I_{m, q} \otimes I_{p} \otimes I_{r s}\right)(\operatorname{vec} A \otimes \operatorname{vec} B \otimes \operatorname{vec} C) \\
= & \left(I_{n q} \otimes I_{m p, s} \otimes I_{r}\right)\left(I_{n} \otimes I_{m, q} \otimes I_{p r s}\right)(\operatorname{vec} A \otimes \operatorname{vec} B \otimes \operatorname{vec} C) .
\end{aligned}
$$

Since $A, B$ and $C$ be arbitrary,

$$
I_{m n q} \otimes I_{p, s} \otimes I_{r}=I_{n} \otimes I_{m, q} \otimes I_{p r s}
$$

using (1), (2) and (3).

Theorem 3.2. The following equalities hold.

$$
\begin{aligned}
& \left(I_{n} \otimes I_{m, q s v} \otimes I_{p r u}\right)\left(I_{m n q} \otimes I_{p, s v} \otimes I_{r u}\right)\left(I_{m n p q s} \otimes I_{r, v} \otimes I_{u}\right) \\
& =\left(I_{n q} \otimes I_{m p, s v} \otimes I_{r u}\right)\left(I_{n} \otimes I_{m, q} \otimes I_{p r u s v}\right)\left(I_{m n p q s} \otimes I_{r, v} \otimes I_{u}\right) \\
& =\left(I_{n} \otimes I_{m, q s v} \otimes I_{p r u}\right)\left(I_{m n q s} \otimes I_{p r, v} \otimes I_{u}\right)\left(I_{m n q} \otimes I_{p, s} \otimes I_{r u v}\right) \\
& =\left(I_{n q s} \otimes I_{m p r, v} \otimes I_{u}\right)\left(I_{n} \otimes I_{m, q s} \otimes I_{p r u v}\right)\left(I_{m n q} \otimes I_{p, s} \otimes I_{r u v}\right) \\
& =\left(I_{n q} \otimes I_{m p, s v} \otimes I_{r u}\right)\left(I_{m n p q s} \otimes I_{r, v} \otimes I_{u}\right)\left(I_{n} \otimes I_{m, q} \otimes I_{p r s u v}\right) \\
& =\left(I_{n q s} \otimes I_{m p r, v} \otimes I_{u}\right)\left(I_{n q} \otimes I_{m p, s} \otimes I_{r u v}\right)\left(I_{n} \otimes I_{m, q} \otimes I_{p r s u v}\right) \\
& =\left(I_{n q} \otimes I_{m p, s v} \otimes I_{r u}\right)\left(I_{n} \otimes I_{m, q} \otimes I_{p s} \otimes I_{r, v} \otimes I_{u}\right)
\end{aligned}
$$

Proof. Let $A: m \times n, B: p \times q, C: r \times s$ and $D: u \times v$ be arbitrary matrices. 
$[1]$

$$
\begin{aligned}
& \operatorname{vec}(A \otimes B \otimes C \otimes D) \\
=\quad & \operatorname{vec}(A \otimes B \otimes(C \otimes D)) \\
=\quad & \left(I_{n} \otimes I_{m, q s v} \otimes I_{p r u}\right)\left(I_{m n q} \otimes I_{p, s v} \otimes I_{r u}\right)(\operatorname{vec} A \otimes \operatorname{vec} B \otimes \operatorname{vec}(C \otimes D)) \\
=\quad & \left(I_{n} \otimes I_{m, q s v} \otimes I_{p r u}\right)\left(I_{m n q} \otimes I_{p, s v} \otimes I_{r u}\right) \\
& \quad \cdot\left(\left(I_{m n p q}(\operatorname{vec} A \otimes \operatorname{vec} B)\right) \otimes\left(\left(I_{s} \otimes I_{r, v} \otimes I_{u}\right)(\operatorname{vec} C \otimes \operatorname{vec} D)\right)\right. \\
=\quad & \left(I_{n} \otimes I_{m, q s v} \otimes I_{p r u}\right)\left(I_{m n q} \otimes I_{p, s v} \otimes I_{r u}\right) \\
= & \quad\left(I_{n} \otimes I_{m, q s v} \otimes I_{p r u}\right)\left(I_{m n q} \otimes I_{p, s v} \otimes I_{r u}\right)\left(I_{m n p q s} \otimes I_{r, v} \otimes I_{u}\right) \\
& \quad \cdot(\operatorname{vec} A \otimes \operatorname{vec} B \otimes \operatorname{vec} C \otimes \operatorname{vec} D),
\end{aligned}
$$

using (6), (4), (5), (1) and (2).

[2]

$$
\begin{aligned}
& \operatorname{vec}(A \otimes B \otimes C \otimes D) \\
& =\operatorname{vec}(A \otimes B \otimes(C \otimes D)) \\
& =\left(I_{n q} \otimes I_{m p, s v} \otimes I_{r u}\right)\left(I_{n} \otimes I_{m, q} \otimes I_{\text {prusv }}\right)(\operatorname{vec} A \otimes \operatorname{vec} B \otimes \operatorname{vec}(C \otimes D)) \\
& =\left(I_{n q} \otimes I_{m p, s v} \otimes I_{r u}\right)\left(I_{n} \otimes I_{m, q} \otimes I_{\text {prusv }}\right) \\
& \cdot\left(I_{m n p q}(\operatorname{vec} A \otimes \operatorname{vec} B)\right) \otimes\left(\left(I_{s} \otimes I_{r, v} \otimes I_{u}\right)(\operatorname{vec} C \otimes \operatorname{vec} D)\right) \\
& =\left(I_{n q} \otimes I_{m p, s v} \otimes I_{r u}\right)\left(I_{n} \otimes I_{m, q} \otimes I_{p r u s v}\right)\left(I_{m n p q s} \otimes I_{r, v} \otimes I_{u}\right) \\
& \text { · }(\operatorname{vec} A \otimes \operatorname{vec} B \otimes \operatorname{vec} C \otimes \operatorname{vec} D) \text {, }
\end{aligned}
$$

using (7), (4), (5), (1) and (2).

[3]

$$
\begin{aligned}
& \operatorname{vec}(A \otimes B \otimes C \otimes D) \\
=\quad & \operatorname{vec}(A \otimes(B \otimes C) \otimes D) \\
=\quad & \left(I_{n} \otimes I_{m, q s v} \otimes I_{p r u}\right)\left(I_{m n q s} \otimes I_{p r, v} \otimes I_{u}\right)(\operatorname{vec} A \otimes \operatorname{vec}(B \otimes C) \otimes \operatorname{vec} D) \\
= & \left(I_{n} \otimes I_{m, q s v} \otimes I_{p r u}\right)\left(I_{m n q s} \otimes I_{p r, v} \otimes I_{u}\right) \\
& \quad \cdot\left(\left(I_{m n} \operatorname{vec} A\right) \otimes\left(\left(I_{q} \otimes I_{p, s} \otimes I_{r}\right)(\operatorname{vec} B \otimes \operatorname{vec} C)\right) \otimes\left(I_{u v} \operatorname{vec} D\right)\right) \\
=\quad & \left(I_{n} \otimes I_{m, q s v} \otimes I_{p r u}\right)\left(I_{m n q s} \otimes I_{p r, v} \otimes I_{u}\right) \\
= & \quad\left(I_{n} \otimes I_{m, q s v} \otimes I_{p r u}\right)\left(I_{m n q s} \otimes I_{p r, v} \otimes I_{u}\right)\left(I_{m n q} \otimes I_{p, s} \otimes I_{r u v}\right) \\
& \quad \cdot(\operatorname{vec} A \otimes \operatorname{vec} B \otimes \operatorname{vec} C \otimes \operatorname{vec} D),
\end{aligned}
$$

using (6), (5), (4), (1) and (2). 
$[4]$

$$
\begin{aligned}
& \operatorname{vec}(A \otimes B \otimes C \otimes D) \\
= & \operatorname{vec}(A \otimes(B \otimes C) \otimes D) \\
=\quad & \left(I_{n q s} \otimes I_{m p r, v} \otimes I_{u}\right)\left(I_{n} \otimes I_{m, q s} \otimes I_{\text {pruv }}\right)(\operatorname{vec} A \otimes \operatorname{vec}(B \otimes C) \otimes \operatorname{vec} D) \\
= & \left(I_{n q s} \otimes I_{m p r, v} \otimes I_{u}\right)\left(I_{n} \otimes I_{m, q s} \otimes I_{\text {pruv }}\right) \\
& \cdot\left(\left(I_{m n} \operatorname{vec} A\right) \otimes\left(\left(I_{q} \otimes I_{p, s} \otimes I_{r}\right)(\operatorname{vec} B \otimes \operatorname{vec} C)\right) \otimes\left(I_{u v} \operatorname{vec} D\right)\right) \\
=\quad & \left(I_{n q s} \otimes I_{m p r, v} \otimes I_{u}\right)\left(I_{n} \otimes I_{m, q s} \otimes I_{p r u v}\right) \\
= & \left(I_{n q s} \otimes I_{m p r, v} \otimes I_{u}\right)\left(I_{n} \otimes I_{m, q s} \otimes I_{\text {pruv }}\right)\left(I_{m n q} \otimes I_{p, s} \otimes I_{r u v}\right) \\
& \quad \cdot(\operatorname{vec} A \otimes \operatorname{vec} B \otimes \operatorname{vec} C \otimes \operatorname{vec} D),
\end{aligned}
$$

using (7), (5), (4), (1) and (2).

[5]

$$
\begin{aligned}
& \operatorname{vec}(A \otimes B \otimes C \otimes D) \\
= & \operatorname{vec}((A \otimes B) \otimes C \otimes D) \\
=\quad & \left(I_{n q} \otimes I_{m p, s v} \otimes I_{r u}\right)\left(I_{m n p q s} \otimes I_{r, v} \otimes I_{u}\right)(\operatorname{vec}(A \otimes B) \otimes \operatorname{vec} C \otimes \operatorname{vec} D) \\
=\quad & \left(I_{n q} \otimes I_{m p, s v} \otimes I_{r u}\right)\left(I_{m n p q s} \otimes I_{r, v} \otimes I_{u}\right) \\
& \cdot\left(\left(\left(I_{n} \otimes I_{m, q} \otimes I_{p}\right)(\operatorname{vec} A \otimes \operatorname{vec} B)\right) \otimes\left(I_{r s} \operatorname{vec} C\right) \otimes\left(I_{u v} \operatorname{vec} D\right)\right) \\
=\quad & \left(I_{n q} \otimes I_{m p, s v} \otimes I_{r u}\right)\left(I_{m n p q s} \otimes I_{r, v} \otimes I_{u}\right) \\
& \quad \cdot\left(I_{n} \otimes I_{m, q} \otimes I_{p} \otimes I_{r s} \otimes I_{u v}\right)(\operatorname{vec} A \otimes \operatorname{vec} B \otimes \operatorname{vec} C \otimes \operatorname{vec} D) \\
=\quad & \left(I_{n q} \otimes I_{m p, s v} \otimes I_{r u}\right)\left(I_{m n p q s} \otimes I_{r, v} \otimes I_{u}\right)\left(I_{n} \otimes I_{m, q} \otimes I_{p r s u v}\right)
\end{aligned}
$$

using (6), (5), (8), (1) and (2).

[6]

$$
\begin{aligned}
& \operatorname{vec}(A \otimes B \otimes C \otimes D) \\
= & \operatorname{vec}((A \otimes B) \otimes C \otimes D) \\
=\quad & \left(I_{n q s} \otimes I_{m p r, v} \otimes I_{u}\right)\left(I_{n q} \otimes I_{m p, s} \otimes I_{r u v}\right)(\operatorname{vec}(A \otimes B) \otimes \operatorname{vec} C \otimes \operatorname{vec} D) \\
=\quad & \left(I_{n q s} \otimes I_{m p r, v} \otimes I_{u}\right)\left(I_{n q} \otimes I_{m p, s} \otimes I_{r u v}\right) \\
& \quad \cdot\left(\left(\left(I_{n} \otimes I_{m, q} \otimes I_{p}\right)(\operatorname{vec} A \otimes \operatorname{vec} B)\right) \otimes\left(I_{r s} \operatorname{vec} C\right) \otimes\left(I_{u v} \operatorname{vec} D\right)\right) \\
=\quad & \left(I_{n q s} \otimes I_{m p r, v} \otimes I_{u}\right)\left(I_{n q} \otimes I_{m p, s} \otimes I_{r u v}\right)\left(I_{n} \otimes I_{m, q} \otimes I_{p r s u v}\right) \\
& \quad(\operatorname{vec} A \otimes \operatorname{vec} B \otimes \operatorname{vec} C \otimes \operatorname{vec} D),
\end{aligned}
$$

using (7), (5), (4), (1) and (2). 
$[7]$

$$
\begin{aligned}
& \operatorname{vec}(A \otimes B \otimes C \otimes D) \\
= & \operatorname{vec}((A \otimes B) \otimes(C \otimes D)) \\
= & \left(I_{n q} \otimes I_{m p, s v} \otimes I_{r u}\right)(\operatorname{vec}(A \otimes B) \otimes \operatorname{vec}(C \otimes D)) \\
= & \left(I_{n q} \otimes I_{m p, s v} \otimes I_{r u}\right) \\
& \cdot\left(\left(I_{n} \otimes I_{m, q} \otimes I_{p}\right)(\operatorname{vec} A \otimes \operatorname{vec} B) \otimes\left(I_{s} \otimes I_{r, v} \otimes I_{u}\right)(\operatorname{vec} C \otimes \operatorname{vec} D)\right) \\
= & \left(I_{n q} \otimes I_{m p, s v} \otimes I_{r u}\right)\left(I_{n} \otimes I_{m, q} \otimes I_{p s} \otimes I_{r, v} \otimes I_{u}\right) \\
& \quad \cdot(\operatorname{vec} A \otimes \operatorname{vec} B \otimes \operatorname{vec} C \otimes \operatorname{vec} D),
\end{aligned}
$$

using (4), (5), (1) and (2).

Theorem 3.3. The following equalities hold.

$$
\begin{gathered}
\left(I_{n} \otimes I_{m, q s v} \otimes I_{p r u}\right)\left(I_{m n q} \otimes I_{p, s v} \otimes I_{r u}\right)=\left(I_{n q} \otimes I_{m p, s v} \otimes I_{r u}\right)\left(I_{n} \otimes I_{m, q} \otimes I_{p r u s v}\right) \\
\left(I_{n} \otimes I_{m, q s v} \otimes I_{p r u}\right)\left(I_{m n q s} \otimes I_{p r, v} \otimes I_{u}\right)=\left(I_{n q s} \otimes I_{m p r, v} \otimes I_{u}\right)\left(I_{n} \otimes I_{m, q s} \otimes I_{p r u v}\right) \\
\left(I_{n q} \otimes I_{m p, s v} \otimes I_{r u}\right)\left(I_{m n p q s} \otimes I_{r, v} \otimes I_{u}\right)=\left(I_{n q s} \otimes I_{m p r, v} \otimes I_{u}\right)\left(I_{n q} \otimes I_{m p, s} \otimes I_{r u v}\right) \\
\left(I_{m n p q s} \otimes I_{r, v} \otimes I_{u}\right)\left(I_{n} \otimes I_{m, q} \otimes I_{\text {prsuv }}\right)=I_{n} \otimes I_{m, q} \otimes I_{p s} \otimes I_{r, v} \otimes I_{u}
\end{gathered}
$$

Proof. Using results on Theorem 3.2, we can show easily that the above equalities hold.

Corollary 3.4. The following equalities hold.

$$
\begin{aligned}
& \left(I_{n} \otimes I_{m, n^{3}} \otimes I_{m^{3}}\right)\left(I_{m n^{2}} \otimes I_{m, n^{2}} \otimes I_{m^{2}}\right)\left(I_{m^{2} n^{3}} \otimes I_{m, n} \otimes I_{m}\right) \\
& =\left(I_{n^{2}} \otimes I_{m^{2}, n^{2}} \otimes I_{m^{2}}\right)\left(I_{n} \otimes I_{m, n} \otimes I_{m^{3} n^{2}}\right)\left(I_{m^{2} n^{3}} \otimes I_{m, n} \otimes I_{m}\right) \\
& =\left(I_{n} \otimes I_{m, n^{3}} \otimes I_{m^{3}}\right)\left(I_{m n^{3}} \otimes I_{m^{2}, n} \otimes I_{m}\right)\left(I_{m n^{2}} \otimes I_{m, n} \otimes I_{m^{2} n}\right) \\
& =\left(I_{n^{3}} \otimes I_{m^{3}, n} \otimes I_{m}\right)\left(I_{n} \otimes I_{m, n^{2}} \otimes I_{m^{3} n}\right)\left(I_{m n^{2}} \otimes I_{m, n} \otimes I_{m^{2} n}\right) \\
& =\left(I_{n^{2}} \otimes I_{m^{2}, n^{2}} \otimes I_{m^{2}}\right)\left(I_{m^{2} n^{3}} \otimes I_{m, n} \otimes I_{m}\right)\left(I_{n} \otimes I_{m, n} \otimes I_{m^{3} n^{2}}\right) \\
& =\left(I_{n^{3}} \otimes I_{m^{3}, n} \otimes I_{m}\right)\left(I_{n^{2}} \otimes I_{m^{2}, n} \otimes I_{m^{2} n}\right)\left(I_{n} \otimes I_{m, n} \otimes I_{m^{3} n^{2}}\right) \\
& =\left(I_{n^{2}} \otimes I_{m^{2}, n^{2}} \otimes I_{m^{2}}\right)\left(I_{n} \otimes I_{m, n} \otimes I_{m n} \otimes I_{m, n} \otimes I_{m}\right)
\end{aligned}
$$

Proof. Let $A, B, C$ and $D$ be arbitrary $m \times n$ matrices. Substituting $p=$ $r=u=m, q=s=v=n$ and applying Theorem 3.2, we get the following relations. 
$[1]$

$$
\begin{aligned}
\operatorname{vec}(A \otimes B \otimes C \otimes D) & \\
=\quad\left(I_{n} \otimes I_{m, n^{3}} \otimes I_{m^{3}}\right)\left(I_{m n^{2}} \otimes\right. & \left.I_{m, n^{2}} \otimes I_{m^{2}}\right)\left(I_{m^{2} n^{3}} \otimes I_{m, n} \otimes I_{m}\right) \\
& \cdot(\operatorname{vec} A \otimes \operatorname{vec} B \otimes \operatorname{vec} C \otimes \operatorname{vec} D) .
\end{aligned}
$$

$[2]$

$$
\begin{aligned}
& \operatorname{vec}(A \otimes B \otimes C \otimes D) \\
=\quad\left(I_{n^{2}} \otimes I_{m^{2}, n^{2}} \otimes I_{m^{2}}\right)\left(I_{n} \otimes\right. & \left.I_{m, n} \otimes I_{m^{3} n^{2}}\right)\left(I_{m^{2} n^{3}} \otimes I_{m, n} \otimes I_{m}\right) \\
& \cdot(\operatorname{vec} A \otimes \operatorname{vec} B \otimes \operatorname{vec} C \otimes \operatorname{vec} D) .
\end{aligned}
$$

$[3]$

$$
\begin{aligned}
& \operatorname{vec}(A \otimes B \otimes C \otimes D) \\
=\quad\left(I_{n} \otimes I_{m, n^{3}} \otimes I_{m^{3}}\right)\left(I_{m n^{3}} \otimes\right. & \left.I_{m^{2}, n} \otimes I_{m}\right)\left(I_{m n^{2}} \otimes I_{m, n} \otimes I_{m^{2} n}\right) \\
& \cdot(\operatorname{vec} A \otimes \operatorname{vec} B \otimes \operatorname{vec} C \otimes \operatorname{vec} D) .
\end{aligned}
$$

$[4]$

$$
\begin{aligned}
& \operatorname{vec}(A \otimes B \otimes C \otimes D) \\
=\quad\left(I_{n^{3}} \otimes I_{m^{3}, n} \otimes I_{m}\right)\left(I_{n} \otimes\right. & \left.I_{m, n^{2}} \otimes I_{m^{3} n}\right)\left(I_{m n^{2}} \otimes I_{m, n} \otimes I_{m^{2} n}\right) \\
& \cdot(\operatorname{vec} A \otimes \operatorname{vec} B \otimes \operatorname{vec} C \otimes \operatorname{vec} D) .
\end{aligned}
$$

$[5]$

$$
\begin{aligned}
& \operatorname{vec}(A \otimes B \otimes C \otimes D) \\
=\quad\left(I_{n^{3}} \otimes I_{m^{3}, n} \otimes I_{m}\right)\left(I_{n^{2}} \otimes\right. & \left.I_{m^{2}, n} \otimes I_{m^{2} n}\right)\left(I_{n} \otimes I_{m, n} \otimes I_{m^{3} n^{2}}\right) \\
& \cdot(\operatorname{vec} A \otimes \operatorname{vec} B \otimes \operatorname{vec} C \otimes \operatorname{vec} D) .
\end{aligned}
$$

[6]

$$
\begin{aligned}
& \operatorname{vec}(A \otimes B \otimes C \otimes D) \\
=\quad\left(I_{n^{3}} \otimes I_{m^{3}, n} \otimes I_{m}\right)\left(I_{n^{2}} \otimes I_{m^{2}, n} \otimes\right. & \left.I_{m^{2} n}\right)\left(I_{n} \otimes I_{m, n} \otimes I_{m^{3} n^{2}}\right) \\
& \cdot(\operatorname{vec} A \otimes \operatorname{vec} B \otimes \operatorname{vec} C \otimes \operatorname{vec} D) .
\end{aligned}
$$

[7]

$$
\begin{aligned}
& \operatorname{vec}(A \otimes B \otimes C \otimes D) \\
=\quad\left(I_{n^{2}} \otimes I_{m^{2}, n^{2}} \otimes I_{m^{2}}\right)\left(I_{n} \otimes\right. & \left.I_{m, n} \otimes I_{m n} \otimes I_{m, n} \otimes I_{m}\right) \\
& \cdot(\operatorname{vec} A \otimes \operatorname{vec} B \otimes \operatorname{vec} C \otimes \operatorname{vec} D) .
\end{aligned}
$$


Corollary 3.5. The following equalities hold.

$$
\begin{aligned}
& \left(I_{n} \otimes I_{m, n^{3}} \otimes I_{m^{3}}\right)\left(I_{m n^{2}} \otimes I_{m, n^{2}} \otimes I_{m^{2}}\right)=\left(I_{n^{2}} \otimes I_{m^{2}, n^{2}} \otimes I_{m^{2}}\right)\left(I_{n} \otimes I_{m, n} \otimes I_{m^{3} n^{2}}\right) \\
& \left(I_{n} \otimes I_{m, n^{3}} \otimes I_{m^{3}}\right)\left(I_{m n^{3}} \otimes I_{m^{2}, n} \otimes I_{m}\right)=\left(I_{n^{3}} \otimes I_{m^{3}, n} \otimes I_{m}\right)\left(I_{n} \otimes I_{m, n^{2}} \otimes I_{m^{3} n}\right) \\
& \left(I_{n^{2}} \otimes I_{m^{2}, n^{2}} \otimes I_{m^{2}}\right)\left(I_{m^{3} n^{2}} \otimes I_{m, n} \otimes I_{m}\right)=\left(I_{n^{3}} \otimes I_{m^{3}, n} \otimes I_{m}\right)\left(I_{n^{2}} \otimes I_{m^{2}, n} \otimes I_{m^{2} n}\right) \\
& \left(I_{m^{2} n^{3}} \otimes I_{m, n} \otimes I_{m}\right)\left(I_{n} \otimes I_{m, n} \otimes I_{m^{3} n^{2}}\right)=I_{n} \otimes I_{m, n} \otimes I_{m n} \otimes I_{m, n} \otimes I_{m}
\end{aligned}
$$

Proof. Using results on Corollary 3.4, we can show easily that the above equalities is true.

Corollary 3.6. The following equalities hold.

$$
\begin{aligned}
& \left(I_{n} \otimes I_{n, n^{3}} \otimes I_{n^{3}}\right)\left(I_{n^{3}} \otimes I_{n, n^{2}} \otimes I_{n^{2}}\right)\left(I_{n^{5}} \otimes I_{n, n} \otimes I_{n}\right) \\
& =\left(I_{n^{2}} \otimes I_{n^{2}, n^{2}} \otimes I_{n^{2}}\right)\left(I_{n} \otimes I_{n, n} \otimes I_{n^{5}}\right)\left(I_{n^{5}} \otimes I_{n, n} \otimes I_{n}\right) \\
& =\left(I_{n} \otimes I_{n, n^{3}} \otimes I_{n^{3}}\right)\left(I_{n^{4}} \otimes I_{n^{2}, n} \otimes I_{n}\right)\left(I_{n^{3}} \otimes I_{n, n} \otimes I_{n^{3}}\right) \\
& =\left(I_{n^{3}} \otimes I_{n^{3}, n} \otimes I_{n}\right)\left(I_{n} \otimes I_{n, n^{2}} \otimes I_{n^{4}}\right)\left(I_{n^{3}} \otimes I_{n, n} \otimes I_{n^{3}}\right) \\
& =\left(I_{n^{2}} \otimes I_{n^{2}, n^{2}} \otimes I_{n^{2}}\right)\left(I_{n^{5}} \otimes I_{n, n} \otimes I_{n}\right)\left(I_{n} \otimes I_{n, n} \otimes I_{n^{5}}\right) \\
& =\left(I_{n^{3}} \otimes I_{n^{3}, n} \otimes I_{n}\right)\left(I_{n^{2}} \otimes I_{n^{2}, n} \otimes I_{n^{3}}\right)\left(I_{n} \otimes I_{n, n} \otimes I_{n^{5}}\right) \\
& =\left(I_{n^{2}} \otimes I_{n^{2}, n^{2}} \otimes I_{n^{2}}\right)\left(I_{n} \otimes I_{n, n} \otimes I_{n^{2}} \otimes I_{n, n} \otimes I_{n}\right)
\end{aligned}
$$

Proof. Let $A, B, C$ and $D$ be arbitrary $n \times n$ matrices. Substituting $m=p=$ $q=r=s=u=v=n$ and applying Theorem 3.2, Corollary 3.6 is obtained.

$[1]$

$$
\begin{aligned}
& \operatorname{vec}(A \otimes B \otimes C \otimes D) \\
= & \left(I_{n} \otimes I_{n, n^{3}} \otimes I_{n^{3}}\right)\left(I_{n^{3}} \otimes I_{n, n^{2}} \otimes I_{n^{2}}\right)\left(I_{n^{5}} \otimes I_{n, n} \otimes I_{n}\right)
\end{aligned}
$$

$\cdot(\operatorname{vec} A \otimes \operatorname{vec} B \otimes \operatorname{vec} C \otimes \operatorname{vec} D)$.

$[2]$

$$
\begin{aligned}
& \operatorname{vec}(A \otimes B \otimes C \otimes D) \\
= & \left(I_{n^{2}} \otimes I_{n^{2}, n^{2}} \otimes I_{n^{2}}\right)\left(I_{n} \otimes I_{n, n} \otimes I_{n^{5}}\right)\left(I_{n^{5}} \otimes I_{n, n} \otimes I_{n}\right)
\end{aligned}
$$

$\cdot(\operatorname{vec} A \otimes \operatorname{vec} B \otimes \operatorname{vec} C \otimes \operatorname{vec} D)$.

$[3]$

$$
\begin{aligned}
& \operatorname{vec}(A \otimes B \otimes C \otimes D) \\
=\quad\left(I_{n} \otimes I_{n, n^{3}} \otimes I_{n^{3}}\right)\left(I_{n^{4}} \otimes\right. & \left.I_{n^{2}, n} \otimes I_{n}\right)\left(I_{n^{3}} \otimes I_{n, n} \otimes I_{n^{3}}\right) \\
& \quad \cdot(\operatorname{vec} A \otimes \operatorname{vec} B \otimes \operatorname{vec} C \otimes \operatorname{vec} D) .
\end{aligned}
$$


$[4]$

$$
\begin{aligned}
& \operatorname{vec}(A \otimes B \otimes C \otimes D) \\
&=\quad\left(I_{n^{3}} \otimes I_{n^{3}, n} \otimes I_{n}\right)\left(I_{n} \otimes I_{n, n^{2}} \otimes I_{n^{4}}\right)\left(I_{n^{3}} \otimes I_{n, n} \otimes I_{n^{3}}\right) \\
& \quad \cdot(\operatorname{vec} A \otimes \operatorname{vec} B \otimes \operatorname{vec} C \otimes \operatorname{vec} D) .
\end{aligned}
$$

$[5]$

$$
\begin{aligned}
& \operatorname{vec}(A \otimes B \otimes C \otimes D) \\
=\quad\left(I_{n^{3}} \otimes I_{n^{3}, n} \otimes I_{n}\right)\left(I_{n^{2}} \otimes\right. & \left.I_{n^{2}, n} \otimes I_{n^{3}}\right)\left(I_{n} \otimes I_{n, n} \otimes I_{n^{5}}\right) \\
& \quad \cdot(\operatorname{vec} A \otimes \operatorname{vec} B \otimes \operatorname{vec} C \otimes \operatorname{vec} D) .
\end{aligned}
$$

$[6]$

$$
\begin{aligned}
& \operatorname{vec}(A \otimes B \otimes C \otimes D) \\
=\quad\left(I_{n^{3}} \otimes I_{n^{3}, n} \otimes I_{n}\right)\left(I_{n^{2}} \otimes I_{n^{2}, n} \otimes\right. & \left.I_{n^{3}}\right)\left(I_{n} \otimes I_{n, n} \otimes I_{n^{5}}\right) \\
& \cdot(\operatorname{vec} A \otimes \operatorname{vec} B \otimes \operatorname{vec} C \otimes \operatorname{vec} D) .
\end{aligned}
$$

$[7]$

$$
\begin{aligned}
& \operatorname{vec}(A \otimes B \otimes C \otimes D) \\
=\quad\left(I_{n^{2}} \otimes I_{n^{2}, n^{2}} \otimes I_{n^{2}}\right)\left(I_{n} \otimes\right. & \left.I_{n, n} \otimes I_{n^{2}} \otimes I_{n, n} \otimes I_{n}\right) \\
& \quad(\operatorname{vec} A \otimes \operatorname{vec} B \otimes \operatorname{vec} C \otimes \operatorname{vec} D) .
\end{aligned}
$$

Corollary 3.7. The following equalities hold.

$$
\begin{gathered}
\left(I_{n} \otimes I_{n, n^{3}} \otimes I_{n^{3}}\right)\left(I_{n^{3}} \otimes I_{n, n^{2}} \otimes I_{n^{2}}\right)=\left(I_{n^{2}} \otimes I_{n^{2}, n^{2}} \otimes I_{n^{2}}\right)\left(I_{n} \otimes I_{n, n} \otimes I_{n^{5}}\right) \\
\left(I_{n} \otimes I_{n, n^{3}} \otimes I_{n^{3}}\right)\left(I_{n^{4}} \otimes I_{n^{2}, n} \otimes I_{n}\right)=\left(I_{n^{3}} \otimes I_{n^{3}, n} \otimes I_{n}\right)\left(I_{n} \otimes I_{n, n^{2}} \otimes I_{n^{4}}\right) \\
\left(I_{n^{2}} \otimes I_{n^{2}, n^{2}} \otimes I_{n^{2}}\right)\left(I_{n^{5}} \otimes I_{n, n} \otimes I_{n}\right)=\left(I_{n^{3}} \otimes I_{n^{3}, n} \otimes I_{n}\right)\left(I_{n^{2}} \otimes I_{n^{2}, n} \otimes I_{n^{3}}\right) \\
\left(I_{n^{5}} \otimes I_{n, n} \otimes I_{n}\right)\left(I_{n} \otimes I_{n, n} \otimes I_{n^{5}}\right)=I_{n} \otimes I_{n, n} \otimes I_{n^{2}} \otimes I_{n, n} \otimes I_{n}
\end{gathered}
$$

Proof. Substituting $m=p=q=r=s=u=v=n$ and applying Theorem 3.3, we can prove Corollary 3.7.

\section{Conclusions}

There are a lot of unsolved problems or issues regarding Kronecker products, vec-operator and Commutation matrices. For example, there is no exact formula of the general moment of matrix quadratic form. In fact, only the third moment of matrix quadratic form has been solved. The equalities proven in this paper will contribute to study in this area. 


\section{References}

[1] J. R. Magnus and H. Neudecker, The Commutation Matrix: Some Properties and Applications, Ann. Statist. 7 (1979), no. 2, 381-394.

[2] H. Neudecker and T. Wansbeek, Some Results on Commutation Matrices, with Statistical Applications, Canad. J. Statist. 11 (1983), no. 3, 221-231.

[3] H. Neudecker and T. Wansbeek, Fourth-Order Properties of Normally Distributed Random Matrices, Linear Alg. Appl. 97 (1987), 13-21.

[4] D. S. Tracy and S. A. Sultan, Third Moment of Matrix Quadratic Form, Statist. Prob. Letters 16 (1993), 71-76.

YONG SIK YUN

Department of Mathematics

JEJU NATIONAL UNIVERSITY

102 JEJUDAEHAKNO JEJU-SI

Jeju Special Self-Governing Province, KOREA. 690-756

E-mail address: yunys@jejunu.ac.kr

Chul Kang

Department of Applied Mathematics

HANKYONG NATIONAL UNIVERSiTY

327 Chungang-No AnseOng-Si

KYONGGI-DO, KOREA. 456-749

E-mail address: stat@hknu.ac.kr 